\title{
How mTORC1 senses leucine
}

leucine
depletion
enabled the
binding of
sestrin 2 to
GATOR2

The amino acid leucine regulates several aspects of animal physiology. Leucine effects are largely mediated by mTOR complex 1 (mTORC1), which is a key regulator of cell growth that controls a variety of processes, including protein and lipid synthesis, and autophagy. How mTORC1 senses and integrates multiple environmental signals, including leucine levels, is a subject of intense study, and the identity of the leucine sensor has so far been elusive. Sabatini and colleagues now report that the MTORC1 signalling inhibitor sestrin 2 functions as a leucine sensor for the mTORC1 pathway.

An important site of mTORC1 regulation is the lysosomal surface, where both RAG and RHEB GTPases perform key functions, controlling both the lysosomal localization of

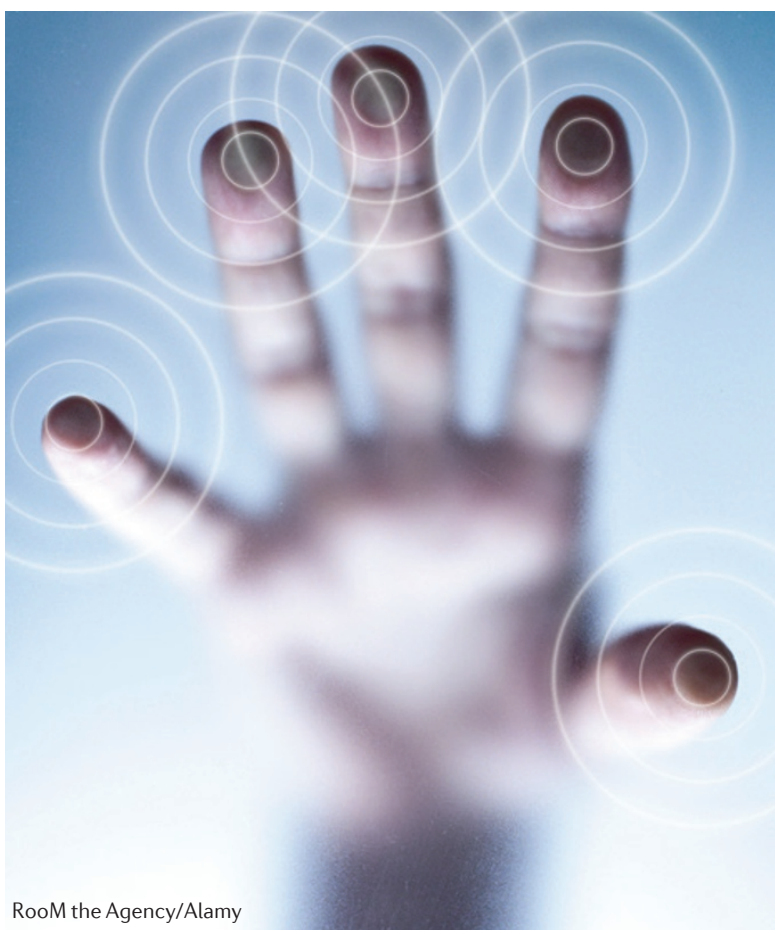

mTORC1 and its activation. Amino acids induce the translocation of mTORC1 to lysosomes in a RAGdependent manner, and the RAGs themselves are subject to complex regulatory mechanisms. GATOR1 (a GTPase activating protein (GAP)) negatively regulates the RAG GTPases, and GATOR2 (an activator of mTORC1 signalling) is a protein complex that interacts with GATOR1. As previous studies have shown that sestrin 2 binds to GATOR2 to inhibit mTORC1 signalling, the authors investigated whether leucine affects the sestrin 2-GATOR2 interaction. Indeed, leucine depletion enabled the binding of sestrin 2 to GATOR2, whereas leucine addition inhibited such binding in human cultured cells. Moreover, leucine disrupted the interaction when added to purified sestrin 2-GATOR2 complexes, indicating that it acts directly on the complex.

To show that leucine binds to sestrin 2 rather than GATOR2, the authors performed an equilibrium binding assay. This binding is specific, as other amino acids (including arginine, which is another important regulator of mTORC1 signalling) were unable to bind to or interacted with sestrin 2 with much lower affinity. Moreover, leucine bound to human sestrin 2 when expressed in bacteria, which do not contain endogenous sestrin or TOR pathways, suggesting that the binding is direct. Importantly, leucine modulated the binding of sestrin 2 to GATOR2 at concentrations similar to those that lead to mTORC1 activation.

Next, the authors tested two mutant forms of sestrin 2: one that bound to leucine but could not bind to GATOR2, and one that was unable to bind to leucine. Expression of either mutant rendered the mTOR pathway leucine-insensitive, demonstrating that sestrin 2 must bind to GATOR2 to inhibit mTORC1 signalling and that it is a leucine sensor that regulates this pathway.

Sestrin 2 is homologous to sestrin 1 and sestrin 3 . Whereas the binding of sestrin 3 to GATOR2 was insensitive to leucine (suggesting that it might be regulated by other signals), sestrin 1 behaved similarly to sestrin 2 . Sestrin 1 thus also seems to be a leucine sensor upstream of mTORC1, although it was not analysed in detail.

Several challenges in the study of mTORC1 signalling remain; for example, it is unknown how the binding of sestrin 2 to GATOR2 leads to mTORC1 suppression. Structural studies are needed to understand how the binding of leucine to sestrin 2 disrupts the interaction with GATOR2, which could help the development of small molecules that either mimic or antagonize the effects of leucine. Such small molecules have important therapeutic potential as, like leucine, they may attenuate the decrease in muscle protein synthesis that is associated with ageing, or may inhibit mTORC1 signalling by having caloric restriction-mimicking properties.

Kim Baumann

ORIGINAL RESEARCH PAPER Wolfson, R. L. et al. Sestrin2 is a leucine sensor for the mTORC1 pathway. Science http://dx.doi.org/10.1126/ science.aab2674 (2015) FURTHER READING Zoncu, R., Efeyan, A. \& Sabatini, D. M. mTOR: from growth signal integration to cancer, diabetes and ageing. Nat. Rev. Mol. Cell Biol. 12, 21-35 (2011) | Shimobayashi, M. \& Hall, M. N. Making new contacts: the mTOR network in metabolism and signalling crosstalk. Nat. Rev. Mol. Cell Biol. 15, 155-162 (2014) 